\title{
Contabilidad Social y Gestión en ONGs*
}

\author{
Fernández Lorenzo, Liliana E.** \\ Geba, Norma E.***
}

\section{Resumen}

La gestión de las Organizaciones no Gubernamentales (ONGs), debe orientarse al cumplimiento de sus objetivos, para lo cual necesita de información adecuada. La información contable económico financiera que emiten regularmente les resulta insuficiente, precisamente por ser entidades no lucrativas por ende, necesitan de información contable con enfoque económico social sobre los impactos que su accionar provoca en el medioambiente interno y externo. El objetivo de este trabajo es identificar y analizar las características de este tipo de información. Metodológicamente ante la imposibilidad de acceder a la información que manejan internamente estos entes, se ha estudiado la contenida en Memorias y Notas a los Balances Tradicionales. Los resultados revelan que esta información carece de homogeneidad, especialmente en cuanto a su cuantificación de sistematicidad, por ello, pierde posibilidad de ser analizada comparativamente y utilizada en la toma de decisiones. Se concluye sobre la importancia del desarrollo de la Contabilidad Social, como especialidad de la disciplina contable y se discuten propuestas de definiciones, conceptos, los modelos de Balance Social ideados y la posibilidad de procesar esta información e integrarla con la económico financiera tradicional.

Palabras clave: Contabilidad Social, Gestión Socioeconómica, Información Contable, Balance Social ONGs.

\section{Recibido: 05-05-03. Aceptado: 05-10-23}

* Trabajo realizado en el marco de proyectos de investigación acreditados y financiados por la Universidad Nacional de La Plata. Argentina

** Profesora de la Facultad de Ciencias Económicas de la Universidad Nacional de La Plata. Directora de proyectos de investigación en el Instituto de Investigaciones y Estudios Contables Autora de libros y artículos sobre investigación contable, contabilidad social, teoría contable. Dirección Postal: Calle 6 entre 47 y 48 Instituto de Investigaciones y Estudios Contables. Of. 418 La Plata, Argentina. E- mail: Ifernan@isis.unip.edu.ar. Autora para correspondencia.

*** Profesora de la Facultad de Ciencias Económicas de la Universidad Nacional de La Plata. Directora de proyectos de investigación en el Instituto de Investigaciones y Estudios Contables Autora de libros y artículos sobre investigación contable, contabilidad social, teoría contable. Dirección Postal: Calle 6 entre 47 y 48 Instituto de Investigaciones y Estudios Contables. Of. 418 La Plata, Argentina. E- mail: normageba@yahoo.com.ar 


\title{
Social Accounting and Management in NGOs
}

\begin{abstract}
The management of non-governmental organizations (NGOs) should be oriented towards fulfilling their objectives, for which adequate information is necessary. The financial economic accounting information that they produce regularly is not sufficient, precisely because they are not lucrative enterprises, and for this reason need accounting information with a social-economic focus as to the impact that their actions produce in the internal and external context. The objective of this paper is to identify and analyze the characteristics of this type of information. Methodologically, due to the impossibility of accessing the information internally managed by these organisms, herein we study the contents of their Annual Reports, notes and traditional balances. The results reveal that this information is not homogeneous, especially in relation to the quantification system, and for this reason it is impossible to analyze and compare the utilization of this information in decision-making processes. The conclusion refers to the importance of developing social accounting as a specialty and to the need to discuss proposed definitions, ideal models of social balance, and the possibility of processing this information and integrating it into a traditional financial form.
\end{abstract}

Key words: Social accounting, social-economic management, accounting information, NGO social balances.

\section{Introducción}

Entre el Sector Público y el Sector Privado tradicional aparece un tercer sector, compuesto por organizaciones que poseen características o atributos de ambos, pero que no son entes mixtos. Este sector adopta diferentes denominaciones, tales como: Organizaciones No Gubernamentales (ONGs), Tercer Sector, Organizaciones de la Sociedad Civil, Sector de la Economía Social y Entidades sin fines de Lucro, etc. Cada denominación pareciera enfatizar determinadas características o variables, algunas de las cuales conllevan toda una ideología.

En los últimos años la participación relativa de este sector se ha acentuado, así como su contribución para resolver problemas sociales. Está integrado por organizaciones cuyas "características comunes más relevantes son: a) Organizaciones formalmente constituidas o con cierto grado de institucionalización, b) Tienen como objetivo la prestación de servicios de interés común para sus asociados y/o la comunidad, sin ánimo de lucro, c) Sus integrantes se adhieren voluntariamente, d) Son autogestionadas por sus miembros, e) Tienen independencia respecto del Estado y e) En caso de distribuir beneficios, lo hacen en función de las personas y no del capital.

Estos rasgos se presentan en diferente grado en las distintas organizaciones que integran este sector. Entre éstas pueden mencionarse como las más relevantes: cooperativas, mutuales, asociaciones, fundaciones, los organismos de segundo y tercer grado que las agrupan, y las redes o uniones de las mismas (Fernández y Geba, 2003). 
El destacado cooperativista y estudioso portugués Da Costa (1984) manifiesta que: "en síntesis en todas estas organizaciones populares la función básica es brindar servicios a sus usuarios y a la comunidad en la que están insertas, también administran la riqueza pero no la acumulan, sino que crean la mencionada propiedad social. Este tercer tipo de propiedad se funda en intereses comunes de los asociados y se vincula con la coyuntura en la que se ubica para sentar las bases de una nueva gestión".

Estas organizaciones, para brindar sus servicios, deben contar con recursos económicos, algunas veces financiados por sus asociados, otras por la comunidad. En ambos casos es necesario contar con una adecuada información para lograr una gestión eficiente y eficaz de los mismos. Pero para la consecución de sus objetivos, que no son precisamente lucrativos, la información económico financiera tradicional se torna insuficiente y debe complementarse con la de tipo económico social.

Para lograr una adecuada gestión socioeconómica, es necesario introducir junto a los objetivos económico-financieros, los relativos a su responsabilidad social asumida tanto para los asociados como para la comunidad. En este aspecto, sigue teniendo vigencia lo expresado hace ya tiempo por García Echevarría (1976), al sostener que hay que tener en cuenta el grado de abstracción y contenido de estos objetivos, para luego hacerlos operativos y que no queden reducidos a meras declaraciones. En todo esto tiene suma trascendencia el sistema de valores vigente en la empresa y en la sociedad donde se inserta.
En consecuencia, la dimensión socioeconómica o económico-social de la realidad, por tratarse de entes económicos, cobra importancia en el sistema de información de las ONGs, tanto hacia dentro como hacia fuera de la organización. La Contabilidad como disciplina social puede contribuir con la obtención y transmisión de conocimientos, metódicos y sistemáticos, principalmente cuantificados, de los impactos sociales producidos en su medioambiente natural y cultural, en el plano socioeconómico. Ello es posible con la incorporación de nuevas variables socioeconómicas en el marco teórico contable, con una acorde ampliación de los sistemas concretos tradicionales y con la emisión de informes contables sociales (internos y externos), a la par de los económico-financieros. Es decir que las ONGs pueden contar con información que, con cierto grado de rigor científico, reúna las características de la buena información para la toma de decisiones, en aras de cumplir con su Responsabilidad Social Asumida y de contribuir al Desarrollo Humano Sustentable.

\section{Información contable y gestión socioeconómica}

Como se considera que las ONGs, al cumplir con sus objetivos, contribuyen directa o indirectamente con el Desarrollo Humano Sustentable, se consideran algunos aspectos de su contenido figurativo. Además se lo relaciona con el rol que muchas veces asumen estas organizaciones y con la posible contribución de la Contabilidad, considerada como disciplina científica social. 
La buena información, metódica y sistemática, preferentemente cuantificada y con cierto grado de homogeneidad, constituye uno de los elementos indispensables para planificary diagnosticar el logro de los objetivos de toda organización. Sobre este tema, se describen algunos atributos que permiten caracterizar a la buena información y las investigaciones realizadas por el equipo -tanto empíricas como teóricas-, relacionadas con la información socioeconómica del denominado Tercer Sector, comentando las conclusiones más significativas.

En estas investigaciones, se analizan los aspectos sustanciales y formales de la información socioeconómica contenida en las Memorias que acompañan a los Estados Contables tradicionales ${ }^{1}$ y en los Informes y Balances Sociales. En cuanto a estos últimos, se comentan las características relevantes de los modelos de balance social ideados en el marco de diferentes proyectos de investigación acreditados ante la Universidad Nacional de La Plata, Argentina. Finalmente se interrelacionan todos estos elementos y se elaboran las conclusiones en aras de mejorar la gestión socioeconómica de las ONGs y en consecuencia, contribuir al logro de un Desarrollo Humano Sustentable.

\subsection{Información y Desarrollo Humano Sustentable}

El concepto de Desarrollo Humano es un término complejo y sobre el cual no siempre existe coincidencia en cuanto a su contenido. En el Informe sobre Desarrollo Humano (Programa de las Naciones Unidas para el Desarrollo -PNUD-, 2000), puede leerse "El desarrollo humano y los derechos humanos se aproximan suficientemente en cuanto a motivaciones y preocupaciones para ser compatibles y congruentes, y son suficientemente diferentes desde el punto de vista de su concepción y estrategia para complementarse entre sí provechosamente. Por consiguiente, la adopción de un criterio más integrado puede brindar mayores recompensas y facilitar en la práctica los esfuerzos comunes por promover la dignidad, el bienestar y la libertad de los individuos en general. Las capacidades pueden variar en forma y contenido, aunque también suelen estar estrechamente relacionadas entre sí. Incluyen, por supuesto, las libertades fundamentales de poder atender a las necesidades corporales, como la capacidad de evitar la inanición y la malnutrición, o para salvarse de la morbilidad prevenible o de la mortalidad prematura. También incluyen las oportunidades habilitadoras que ofrecen, por ejemplo, la educación, o la libertad y los medios económicos para trasladarse libremente y escoger el lugar de residencia. También existen importantes libertades sociales, como la capacidad de participar en la vida de la comunidad, incorporarse al debate público,......El criterio de desarrollo humano se refiere, en última instancia, a todas las capacidades que las personas tengan razones para valorar. El índice de desarrollo humano incorpora las 
capacidades más elementales, como la de vivir una vida larga y saludable, estar bien informado y disfrutar un nivel de vida digno".

Tres años más tarde, el PNUD avanza en este concepto sosteniendo que "Los Objetivos de Desarrollo del Milenio abordan muchos de los reveses más recurrentes del desarrollo humano. Sin embargo, al contrario de los objetivos planteados en los tres primeros Decenios de las Naciones Unidas para el Desarrollo (años 60, 70 y 80), centrados en el crecimiento económico, en estos Objetivos se da prioridad al bienestar de la humanidad y a la reducción de la pobreza... Los objetivos y la promoción de desarrollo humano comparten un ideal común y reflejan el compromiso vital de promover el bienestar de la humanidad, la dignidad, la libertad y la igualdad para todas las personas" (PNUD, 2003).

Pero para el logro de este tan ansiado "desarrollo" es necesario contar con información adecuada. Esta afirmación condice con lo mencionado hace ya tiempo entre las iniciativas de la Cumbre de las Américas sobre Desarrollo Sostenible (1996): “...si bien el desarrollo sostenible y las cuestiones ambientales han pasado a primer plano en lo que toca a planificación y el diseño de Políticas referidas al desarrollo de los países del continente, la base empírica para la adopción de las decisiones sigue siendo notoriamente insuficiente. Los sistemas de contabilidad económica vigentes a nivel nacional y a nivel de sectores, recursos, ecosistemas, instituciones públicas, empresas comerciales, etc. producen una información insuficiente y en la mayoría de los casos inadecuada para abordar los problemas ambientales y de sostenibilidad".

En consecuencia se considera que para el logro de un desarrollo sustentable, tanto a nivel micro como macrosocial, resulta de vital importancia contar con informes sobre los impactos sociales que el accionar de los entes, provocan en su medioambiente interno y externo. En este aspecto la Contabilidad puede llegar a cumplir una función social importante.

"Para que la disciplina contable permita conocer el accionar y los efectos sociales de los entes sobre los recursos naturales y culturales deberá incluir el efecto o impacto, sobre su patrimonio propio y compartido, de otras variables sociales a la par de las económicas, así como sus interrelaciones" (Geba, 2001). Más aún, si la comunidad contable asume como propio el paradigma del Desarrollo Humano Sostenible, aunque esto exija un esfuerzo adicional, estará en mejores condiciones de cumplir "activamente con su responsabilidad social profesional" (Fernández y Geba, 2000a).

\subsection{Las ONGs y su Responsabilidad Social}

En los últimos años la participación relativa de las ONGs se ha acentuado, tanto a nivel nacional como mundial, así como su contribución para resolver problemas sociales. Además y coincidiendo con lo expresado por Ravina (2000), "las distintas formas de cooperación entre las empresas de la economía social y las que pertenecen a otros sectores de la economía, demuestran una renovada posibilidad que se extiende incesantemente".

Coincidiendo con el Foro del Sector Social (Gallup Argentina, 1998), es cierto 
es que "enfrentamos un escenario en el que los roles sociales del Estado, del Mercado y la Sociedad Civil están cambiando", como consecuencia de ello se observa que el Estado delega ciertas prestaciones al Sector Privado y éste, en especial el denominado Tercer Sector, asume en distinto grado determinadas responsabilidades sociales en aras de contribuir a lograr el tan ansiado bien común, es decir que la responsabilidad social de este sector, va más allá de no hacer lo considerado social y legalmente incorrecto en un lugar y tiempo determinados, sino que asume un rol positivo tratando de mejorar la sociedad en la que se inserta. "Se nutre precisamente de su sistema de valores, de su misión, de la filosofía organizacional" (Fernández Lorenzo y Geba, 2000b).

En la mayoría de las ONGs, dicha responsabilidad social forma parte de su finalidad primordial, de su razón de ser, lo asumen como objetivo principal. Para el caso de entidades Cooperativas y Mutuales, está plasmada en lo que se conoce como "Principios Cooperativos" y "Principios Mutuales" que rigen su accionar socialmente responsable.

Para asumir esta responsabilidad concretamente en la práctica, es necesario tomar decisiones socialmente responsables. Se reconoce que esto no es fácil de hacer, pues en ellas inciden una serie de factores: "El desarrollo moral de quien decide; La cultura y la ética de la organización a la que pertenecen; El desarrollo moral de la comunidad en la que se insertan" (Fernández y Geba, 2000b: 21).

Además, este tipo de decisiones que influyen en "la participación social, la producción, el desarrollo no pueden for- mularse en abstracto, sino que requieren de una articulación lógica y adecuada en la que establezcan prioridades y bases. Para lograrlo, es necesario contar con INFORMACIÓN", que permita conocer con qué medios materiales se cuenta, qué servicios se necesitan y quiénes pueden proveerlos (Osorio, 2002).

En el caso de las ONGs, se necesita contar con información socioeconómi$\mathrm{ca}$, que excede a la económico-financiera, y que va a permitir transmitir sus valores y conocer el grado de cumplimiento de la mencionada responsabilidad social asumida.

En otras palabras, teniendo en cuenta la especial naturaleza de las Organizaciones no Lucrativas, el contar y emitir información socioeconómica adquiere un rol muy importante para elevar la calidad del diagnóstico, del análisis, de la toma de decisiones y del control de la situación actual y de su proyección futura. Esto se consigue disponiendo de buena información, que cumpla con determinados requisitos mínimos que la tornen útil para satisfacer las necesidades de sus usuarios.

\subsection{Características de toda buena Información}

La Federación Argentina de Consejos Profesionales de Ciencias Económicas (2002) enuncia una serie de requisito o atributos, para que la información contenida en los estados contables (EECC) pueda ser útil a los usuarios. Entre ellos se destacan:

Pertinencia (atingencia): "La información debe ser apta para satisfacer las necesidades de los usuarios. 
Confiabilidad (Credibilidad): "La información debe ser creíble para sus usuarios, de manera que éstos la acepten". Para ello debe aproximarse a la realidad y ser verificable. En cuanto a la aproximación a la realidad "los estados contables deben presentar descripciones y mediciones que guarden una correspondencia razonable con los fenómenos que pretenden describir"... Para que la información contable se aproxime a la realidad, debe reunir las características siguientes: a) Esencialidad (sustancia sobre forma): ... "las operaciones y los hechos deben contabilizarse y exponerse basándose en su sustancia y realidad ".... económico social, b) Neutralidad (objetividad o ausencia de sesgos): ... "no debe estar sesgada, deformada para favorecer al ente emisor o para influir la conducta de los usuarios hacia alguna dirección particular" y c) Integridad: "La información contenida en los estados contables debe ser completa". En cuanto a la Verificabilidad, para "que la información contable sea confiable, su representatividad debería ser susceptible de comprobación por cualquier persona con pericia suficiente".

Sistematicidad: "La Información contable suministrada debe estar orgánicamente ordenada,"...

Comparabilidad: "La información contenida en los estados contables de un ente debe ser susceptible de comparación con otras informaciones: a) Del mismo ente a la misma fecha o período, b) Del mismo ente a otras fechas o períodos y c) De otros entes".

Claridad (comprensibilidad): "La información debe ser preparada utilizando un lenguaje preciso, que evite las ambigüedades, y que sea inteligible y fácil de comprender por los usuarios que estén dispuestos a estudiarla diligentemente y que tengan un conocimiento razonable". Cuando se menciona el término "Usuarios", a los fines del presente trabajo, se lo considera en su más amplia acepción, es decir no sólo a los inversores sino también usuarios internos al ente como los gerentes.

Las características antes enunciadas, generalmente aplicadas a los Estados Contables Tradicionales, bien pueden ser consideradas para la Emisión de Información Contable Socioeconómica externa a través de los denominados $\mathrm{Ba}$ lances Sociales o adaptarlas a la información socioeconómica interna destinada a la gerencia.

\subsection{Investigaciones empíricas sobre emisión y utilización de información socioeconómica}

En el presente apartado se incluyen las conclusiones a las cuales se arriba en trabajos de investigación realizados sobre Información social emitida por entidades Cooperativas y Mutuales y en otro, la contenida en los denominados Balances Sociales. Los mismos fueron realizados con la finalidad de detectar los niveles de emisión y utilización de la información socioeconómica, así como las características de este tipo de información, en aras de evaluar su utilidad para la toma de decisiones:

a) Información Socioeconómica en Entidades Cooperativas

Con la finalidad de recabar datos referidos entre otros al tema de referencia, se realizó una Investigación empírica en cooperativas de la ciudad de Buenos 
Aires y Gran Buenos Aires (Fernández et al., 2001), utilizando como método de relevamiento de datos una encuesta a informantes claves. La misma tuvo, entre otros objetivos el de evaluar la emisión de información de tipo social y de difundir el modelo de Balance Social Cooperativo Integral ideado (Fernández Lorenzo, et al., 1998). Cabe destacar que: El $56 \%$ de las cooperativas analizadas emiten información social utilizando para ello variados medios, entre ellos se destacan las memorias que acompañan a los estados contables y los principales temas sobre los que informan se refieren a educación, cultura y salud en ese orden.

En función a los datos analizados se concluye que es posible: Que las cooperativas sistematicen la emisión de la información social a través de la aplicación del Balance Social Cooperativo Integral y presentar dicha información en Balance Sociales Consolidados que acumulen la misma y ofrezcan información social a nivel macroeconómico, referida al sector.

b) Información Socioeconómica en Memorias de Entidades Mutuales

La mayoría de las entidades que integran el Sector Social de la Economía, suelen ofrecer información socioeconómica en la Memoria que acompaña a los Estados Contables tradicionales, a través de una síntesis de la actividad desarrollada en el ejercicio y comentarios sobre la situación al cierre y perspectivas futuras del ente. Con la finalidad de verificar empíricamente el tipo y características de esta información se analizan las correspondientes a una muestra de estados contables de entidades mutuales de la ciudad de La Plata, Argentina (Fernández y Geba, 1999).
De este análisis puede destacarse que:

1. Las memorias de las mutuales analizadas, ofrecen comparativamente más información social que económica. Esto pareciera ser una consecuencia de la presentación de la memoria junto a los estados contables tradicionales, cuyo contenido se centra especialmente en aspectos de este último tipo.

2. Se refiere principalmente al pasado y en menor medida al presente. La información proyectada es muy poco significativa. No hacen referencia a planes 0 presupuestos con asignación monetaria para los distintos programas en materia social. En caso de no existir estos últimos, los enunciados podrían transformarse en meras expresiones de deseos.

3. La mayor parte de la información analizada no está cuantificada, y cuando lo está, se utilizan variadas unidades de medida. No se compara con la de ejercicios anteriores (ya sea ésta real o planificada).

De lo antes mencionado se concluye que si bien la memoria que acompaña a los estados contables de las entidades mutuales, parece ser un vehículo a través del cual se transmite información económico social, ésta no es integral, ni metódica ni sistemática. Además con frecuencia no está cuantificada, ni se relaciona con la de ejercicios anteriores, lo que impide realizar un análisis comparativo sobre bases rigurosas y homogéneas.

c) Información Socioeconómica en Entidades Mutuales

Con el objetivo de ahondar en las características de todo tipo de información social emitida por entidades mutuales, se realiza otra investigación empírica 
siguiendo las reglas de la ciencia, presuponiendo que la misma presenta distintas características, en cuanto a forma y contenido, que influyen negativamente en su homogeneidad (Fernández et al., 2003). De un total de 98 mutuales, 41 , es decir el $42 \%$ no se encontraron, de las 57 restantes $(58 \%), 11(19 \%)$ no respondieron y de las $46(81 \%)$ que si respondieron, $3(7 \%)$ no emiten información social y 43 (93\%) si la emiten y todas la utilizan.

Casi toda la población de trabajo le asigna mucha o alguna importancia a informar. Esta circunstancia concuerda con el alto nivel de emisión alcanzado, a pesar de existir en muchos casos, dificultades de tipo financiero para hacerlo.

La información social emitida se concentra principalmente en los asociados, razón de ser de este tipo de entidades, y en los servicios prestados a los mismos. Por lo general trasciende en forma narrativa a través de Boletines Informativos y de la Memoria que acompaña a los Estados Contables Tradicionales. En el primer caso, su emisión es esporádica y en el segundo, con periodicidad anual. Puede intuirse que esto último se debe a la obligatoriedad legal de emitir tales estados.

En un principio, se observan ciertas regularidades en las características de la información emitida, pero profundizando el análisis y en aras de comprobar su homogeneidad, puede observarse que: a) La preeminencia de contenido narrativo y la falta de cuantificación, afecta su objetividad y la posibilidad de compararla y acumularla, b) La información se centra esencialmente sobre el área interna, más precisamente sobre los asociados y en lo referido a servicios prestados, circuns- tancia que le resta riqueza y potencialidad, c) Sólo la mitad incluye información proyectada, dificultando las tareas de gestión, planificación y control sociales y d) La información no se emite conservando siempre una forma o estructura, ni siguiendo una periodicidad regular, salvo en el caso de la Memoria que como ya se dijo, es anual.

En consecuencia, puede concluirse que por lo general, la información social emitida no presenta las características de toda buena información y no es homogénea, disminuyendo su utilidad. A su vez, la información social se emite generalmente con fines de difusión y es poco utilizada para la toma de decisiones y para conocer el grado de cumplimiento de los principios mutuales.

Lo antes dicho, se condice con el hecho de que sólo una entidad emite Balances Sociales, y que son pocas las que conocen algún modelo. El desconocimiento de este tema y de la utilidad potencial de sistematizar la información, quizás explique la escasa homogeneidad de la información social emitida y la baja utilización de la misma a nivel interno.

d) Información socioeconómica en los denominados Balances Sociales

Del estudio cuantitativo realizado por Barbei y Fernández (2002), pueden ofrecerse las siguientes conclusiones:

- Emisión y publicación de Balances Sociales: La mayor cantidad de organizaciones que emiten y publican sus Balances Sociales en la población analizada, corresponde a las privadas no lucrativas (ONGs). La mayor publicación se concentra esencialmente en América del sur, donde se destaca especialmente el caso de 
Colombia, representando algo más de la mitad del total. Si se enfoca este tema en cuanto a la actividad principal del ente emisor, sobresalen los que prestan servicios, item en el que se incluye a la mayoría de las ONGs. Es comprensible que este sector se destaque por su nivel de emisión de información social, ya que sus actividades tienen un estrecho vínculo con lo social y encuentran en los Balances Sociales un medio de informar el real cumplimiento de sus objetivos, obviamente no lucrativos.

- Homogeneidad de la Información Contenida en los Balances Sociales: Esto puede inferirse de la presencia de similares características de la información contenida en ellos. Al respecto puede afirmarse que los balances analizados son heterogéneos sobre todo en cuanto a su sistematicidad y a la unidad de medida que emplean para cuantificar la información. No ocurre lo mismo al analizar las áreas informadas, donde prevalece la externa en un $97 \%$ de los casos; y en las dimensiones informadas y catalogadas como Cultura, Salud y Trabajo (87\%). A estas últimas se las considera Dimensiones Sociales de mayor interés. La mayor homogeneidad se da en cuanto a la referencia temporal. Todos los balances se refieren a información pasada, aunque algunos incluyen también planificaciones o proyecciones. Es conveniente señalar que se considera a la sistematicidad y a la cuantificación, requisitos esenciales para lograr cierta homogeneidad en la información incluida en los Balances Sociales, cosa que hasta el momento no está garantizada.
- Correlación de Variables: Cabe destacar que las organizaciones privadas no lucrativas son las que menos informan sobre Medio Ambiente.

A partir de las observaciones precedentes puede deducirse que la información que integra los Balances Sociales analizados, presenta distintas características, que afectan su homogeneidad, y por ende su comparabilidad. Esto reafirma la necesidad de incrementar la utilidad de los denominados Balances Sociales elaborando modelos de aplicación generalizada, que surjan de un debido proceso en el marco de la Contabilidad Social, como especialidad de la disciplina contable.

\subsection{Modelos de Balance Social para ONGs}

A los fines de este trabajo se entiende como Balance Social: "al informe que emite una organización, cuya finalidad es brindar información metódica y sistemática referida a la responsabilidad social asumida y ejercida por ella. Constituye una herramienta para informar, planificar, evaluar y controlar el ejercicio de dicha responsabilidad. Su conocimiento es de utilidad para directivos, trabajadores, sindicatos, estado, universidades y público en general. En él se encuentran temas concretos que permiten reflexionar y elaborar propuestas para ayudar a concebir y perfeccionar cualquier organización" (Fernández et al., 1998: 21).

Con el objetivo de contribuir con el desarrollo de las ONGs, se elaboraron modelos de aplicación de Balance Social para Entidades Cooperativas (Fernández et al., 1998) y Mutuales (Fernández y 
Geba, 2000b), con un alto grado de homogeneidad entre ellos. Estos modelos: a) Reúnen ciertas características que permiten sistematizar la información aumentado su credibilidad y su aplicabilidad, b) Ofrecen una imagen más acabada del perfil de la entidad al complementar la información económica con la social, c) Incorporan el enfoque de la Contabilidad por Objetivos y d) Cuantifican los datos a través de indicadores socioeconómicos cuali y cuantitativos.

a) Características generales.

Para elaborar estos modelos se parte de considerar que los Principios Cooperativos y Mutuales constituyen un marco común de referencia para evaluar a través de su cumplimiento, la responsabilidad social asumida y ejercida por estas entidades, resaltando su propia identidad (Novarese de Nieto y Fernández, 1995).

Alcance: Los modelos de referencia son aplicables a todo tipo de cooperativas y mutuales, con independencia de la etapa de desarrollo en la que se encuentren. Preferentemente están destinados a las de base o de primer grado, debiendo realizarse adaptaciones cuando las circunstancias así lo requieran. Es de suma importancia que cada entidad ponga de manifiesto sus características distintivas o las circunstancias especiales por las que atraviesa.

Periodicidad: Teniendo en cuenta el tipo de información a suministrar, se considera conveniente su presentación periódica, al finalizar cada ejercicio económico junto a los estados contables legalmente exigidos.

Obligatoriedad: Ante la falta de un tratamiento integral del tema por la legislación vigente en Argentina y la escasa experiencia en cuanto a su implementación práctica, se considera que su presentación en nuestro medio, por lo menos en un principio, debería ser facultativa. Paralelamente, los Organismos de Contralor y las entidades de grado superior, debieran instruir a las entidades de base en cuanto a cómo implementarlo y los beneficios que reporta, entre los que se encuentran: a) La utilidad de contar con información sobre los aportes que las mismas realizan tanto para con sus asociados cuanto para la comunidad y b) La posibilidad de aplicar el modelo en forma sencilla, comenzando con los datos disponibles de manera de incorporar como rutina el procesamiento de la información social a través de su sistema contable.

Una vez superada esta etapa, y para asegurar su emisión generalizada, se propone analizar, para cooperativas y mutuales de gran dimensión, la obligación de presentar un balance social junto a los estados contables tradicionales. Actualmente a través de la Ley de Reforma Laboral argentina se tornan obligatorios para entes que cuenten con más de 300 trabajadores.

Destinatarios: Estos modelos ofrecen información tanto a los agentes sociales internos (directivos, asociados, trabajadores, etc.) como a los externos (clientes, proveedores, federaciones, Estado, y comunidad entre otros). Debe reconocerse que interesa primariamente al área interna. Permite conocer el grado de cumplimiento de los principios cooperativos y mutuales, y de las metas sociales definidas en la planificación socioeconómica anual. Con ello se dispone de un elemento de autosuperación importante que permite evaluar e implementar medi- 
das correctivas, en caso de corresponder. Interesa también a los agentes sociales externos, pues les ofrece una idea más acabada de la organización; a los organismos de segundo y tercer grado, para reforzar su función intercooperati$\mathrm{va} /$ mutual; y al estado para desarrollar sus planes y estrategias sociales.

Contenido: Las cooperativas y mutuales, por su especial naturaleza, constituyen un todo donde se entrelazan lo económico con lo social. Por tal razón, estos modelos de Balance Social combina ambos tipos de información para dar una imagen lo más acabada posible de la misma.

Es recomendable que cada entidad que intente la implementación de un modelo de balance social, organice un sistema de información social integrado con el contable. Este sistema, al procesar adecuadamente los datos, permite obtener información elaborada, sistemática y con control contable.

La simplicidad y claridad conceptual es esencial. Con tal propósito, los indicadores de esta propuesta se exponen a través de magnitudes o cantidades y su relación porcentual con los totales respectivos. Esta información debe presentarse en forma comparativa como mínimo con la del ejercicio inmediato anterior.

b) Enfoque de la Contabilidad por Objetivos:

Resulta necesario que estas entidades, al cierre de cada ejercicio, realicen su planificación socioeconómica y elaboren un presupuesto social para el período siguiente. Esto les permite conocer de antemano las posibilidades con que cuentan y los compromisos que adquieren en el plano social. A esto se denomina "responsabilidad social asumi- da". En su planificación social deben asignar importancia relativa a sus objetivos y metas, para evitar una manipulación a posteriori, una vez obtenidos los resultados. Si no establece prioridades, se interpreta que todos tienen el mismo peso relativo. Las metas deben ser concretas, cuantificadas, realistas, evaluables y alcanzables en el período propuesto. Los programas sociales indican en forma literaria qué se va a realizar, y los presupuestos sociales establecen una asignación monetaria para la ejecución de cada uno de ellos. Con este enfoque es posible que se evalúe su desempeño, comparando las metas propuestas o planificadas con la gestión social desarrollada y los resultados obtenidos.

c) Medición a través de Indicadores Socioeconómicos:

Los modelos propuestos se basan en la medición del cumplimiento de los Principios Cooperativos y Mutuales a través de indicadores socioeconómicos cuantitativos y cualitativos, expresados en diferentes unidades de medida.

Los indicadores propuestos constituyen la información socioeconómica básica que toda entidad debería elaborar y presentar a los fines de posibilitar su comparación. Se seleccionaron los indicadores más relevantes, ya que una información excesiva puede oscurecer lo que se pretende medir y puede obstaculizar su efectiva implementación. No obstante ello, son modelos flexibles ya que es posible complementarlos con indicadores adicionales, siempre que se respete su integridad. En la medida en que esta herramienta acompañe la vida institucional, puede desarrollar mayor complejidad y proyectarse a otros interlocutores sociales. 
Estos modelos combinan información social y económica tanto para el área interna como externa. Constan en esencia de tres informes básicos, que salvando sus diferencias específicas de cada uno, podemos denominar: Estructura Socio laboral, Estado de Situación Social y Estado de Opinión Social, acompañados de Información complementaria y Fundamentos y pautas para su elaboración.

En los dos últimos informes, la información se agrupa de acuerdo a los Principios Cooperativos y Mutuales que guían la vida institucional. Se considera cada principio por separado atendiendo a una necesidad metodológica, pero debe hacerse la salvedad que no son independientes unos de otros. Por ello las entidades no deben ser evaluadas con base en uno de ellos sino por la manera en que adhieren a los mismos como una totalidad.

Los modelos de balance social presentan información socioeconómica en forma sintética, a través de indicadores que se agrupan de la siguiente forma:

Estructura Socio Laboral: Clasifica a los integrantes de las entidades (asociados y trabajadores) según variables socio-demográficas, tales como edad, sexo, nivel de instrucción. Ofrece un marco de referencia para la comprensión del resto de los indicadores.

Estado de Situación Social: Para cada principio cooperativo/mutual se definen aspectos relevantes a medir a través de indicadores. Estos indicadores se basan preferentemente en la información primaria de cada ente (registros contables, libros de actas de asambleas y registros de asistencia a reuniones institucionales entre otros) a fin de asegurar su confiabilidad, objetividad y verificabilidad.
Es recomendable que este Informe sea aprobado por la asamblea anual ordinaria y se exponga junto a los Estados Contables Básicos. Sus destinatarios son tanto internos como externos.

Estado de Opinión Social: Recurre a indicadores cualitativos que resultan necesarios para conocer la opinión de los asociados y trabajadores sobre el cumplimiento de los principios. Muestra aspectos difícilmente cuantificables de otro modo. Estos indicadores se basan en información obtenida a través de encuestas de opinión, en las que los asociados y trabajadores deben responder en forma anónima a un cuestionario. El mismo consta de una serie de preguntas, cada una con cinco alternativas de respuestas posibles. Los encuestados tienen además la posibilidad de presentar sugerencias o propuestas.

La información presentada en este informe está originariamente destinada a los agentes sociales internos por ser de tipo confidencial, siendo decisión de la entidad hacerla pública. Es recomendable que se la analice detenidamente y se intercambien opiniones a fin de proponer mecanismos correctivos en caso de corresponder. Este proceso contribuye a la autosuperación del ente y al afianzamiento de su identidad, aspectos que de lograrse, se reflejarán probablemente en los indicadores objetivos del Estado de Situación Social.

\section{Información complementaria:} Comprende aquella que debe exponerse para mejor interpretar los Informes Socioeconómicos Básicos o, por no estar incluida en ellos, ser necesaria a tales fines. Puede referirse a características especiales de la entidad, del entorno o de 
los hechos informados, situaciones extraordinarias y/o sujetas a acontecimientos futuros y cualquier otra circunstancia que pudiera afectar significativamente la evaluación y toma de decisiones. Forma parte integrante de los informes, se presenta en su encabezamiento y se desarrolla a través de notas, cuadros y anexos, debidamente referenciados.

Es necesario que estos modelos de aplicación surjan de un debido proceso, en el marco de la Contabilidad Social. Para hacer más ágil este proceso se proyecta diseñar un software que, utilizando el proceso contable tradicional, elabore información social y la integre con la económico-financiera.

\subsection{Contabilidad Social y la Teoría General Contable}

Frente al desafío mencionado en el punto anterior, se plantea la importancia del desarrollo de la Contabilidad Social, como especialidad de la disciplina contable, considerada con un enfoque científico. Así entonces se realizan investigaciones teóricas a los fines de delimitar el universo de su discurso en el marco de una teoría general del conocimiento contable.

La Contabilidad ha tenido desde sus orígenes una marcada tradición patrimonialista, con un enfoque eminentemente económico-financiero referido a los Estados Contables para terceros, que actualmente cuenta con un importante desarrollo normativo. Mucho se ha discutido respecto del status epistemológico de la Contabilidad. Distintas posturas existentes la consideran técnica, tecnología, ciencia social y hasta sistema de información. Tal circunstancia parece origi- narse en otorgar distintos contenidos al dominio del discurso contable. Esta falta de consenso ha incidido negativamente en el desarrollo de una Teoría General del Conocimiento Contable, compartida tanto en el ámbito universitario, organismos profesionales y graduados.

La CONTABILIDAD como disciplina, con mayúsculas, estudia la realidad económico social. Si se la describe con un enfoque más amplio y abarcativo que el tradicional, permite incluir las distintas especialidades contables: patrimonial, pública, socio económica, gerencial, etc. Su función social puede describirse esencialmente a través de dos procesos: uno de descripción y otro de comunicación de los impactos económico sociales generados y recibidos por un ente determinado. Cuenta con un bagaje doctrinario, que si bien reclama una Teoría General del Conocimiento Contable, sirve de base para la elaboración de modelos, reglas normativas tecnológicamente controladas, sistemas físicos o concretos e informes.

"Se destaca que "el fin último" de la Contabilidad no es registrar metódicamente algunos hechos de la realidad social (técnica); ni tampoco elaborar o diseñar normas sobre la confección de Estados Contables para resolver cuestiones de hecho (tecnología social); sino valerse de los instrumentos de información elaborados para obtener conocimientos metódicos y sistemáticos de la porción de la realidad considerada. En consecuencia, se la considera como una disciplina científica social, con identidad propia. Así entendida puede brindar un mayor y mejor aporte a la sociedad" (Geba y Fernández, 2002).

Una de las especialidades contables de más reciente data es la denomi- 
nada "Contabilidad Social" (CS). Como aún no se ha logrado consenso en cuanto al nombre que mejor describa e identifique y como tampoco existe uniformidad en cuanto a su contenido, campo de actuación o dominio de su discurso, se plantea una investigación exploratoria sobre sus definiciones y conceptos incluidos en material bibliográfico referido al tema. Se analizan estos aspectos y contribuye en algo a clarificarlos (Fernández y Barbei, 2004). A continuación se comentan algunas de las conclusiones a que se arribó.

"Frente a un mismo hecho o acto generador de impactos sociales, pueden adoptarse distintos enfoques de análisis y así procesar contablemente impactos diferentes. La mayoría de los autores, consideran dentro del dominio del discurso de la Contabilidad Social, a los impactos generados por el ente en el medioambiente. ...Los menos, analizan la incidencia de esos impactos en el patrimonio del ente, es decir utilizan un enfoque patrimonial, aunque hacen referencia a la CS. Según el marco teórico desarrollado en este trabajo, se considera que los primeros si se refieren a Contabilidad Social como especialidad o segmento contable; mientras que los segundos, están en la órbita de la Contabilidad Patrimonial o Financiera y no en la de la CS".

Se señala en el referido informe que en "las definiciones o conceptos analizados se observa que es frecuente la mención de informes sociales como uno de los principales productos del proceso de comunicación, pero estos informes surgen de diferentes procesos y presentan contenidos y formas muy diversas. A todo lo antes dicho se suma, que son escasas las precisiones que realizan los emisores sobre aspectos teóricos de la CS. Esto puede vincularse a que en la evolución de esta especialidad contable, al igual de lo ocurrido con la patrimonial, la práctica, generalmente ha precedido a los desarrollos teóricos".

Al analizar las definiciones los autores observan que los artículos más antiguos profundizan la temática de la Responsabilidad Social y, de manera conexa, con un nivel secundario de importancia, se refieren a la Contabilidad Social. Luego se observa una preocupación mayor en el aspecto práctico que está exteriorizado a través de los Informes Sociales vinculados a la CS...En algunos casos, en los artículos de mediados de la década del 90, se le asigna especial relevancia al tratamiento de la CS en el marco de una estructura general del conocimiento.

Por lo expuesto Fernández y Barbei (2004) plantean "la necesidad de utilizar un lenguaje científico no formal que incluya definiciones nominales precisas, si se pretende contribuir al desarrollo de la Contabilidad Social como especialidad contable y su futura inclusión en una estructura orgánica del conocimiento contable" y caracterizan la Contabilidad social como rama, segmento o especialidad contable los siguientes, precisando los siguientes elementos:

Objeto de estudio: los impactos medioambientales relacionados con un ente.

Objetivo: obtener y brindar un conocimiento metódico y sistemático de estos impactos.

Impacto medioambiental: Es el efecto o variación generado por un ente en su medioambiente. El origen de estos impactos puede deberse: a la mera exis- 
tencia del ente o la actividad desarrollada por el mismo. Comprende tanto sus acciones, inacciones (decisiones de no.hacer) $u$ omisiones.

Medioambiente: Entorno en que se desarrollan los seres vivos que engloba todos los factores que influyen en su desarrollo, excluido el factor genético no manipulado artificialmente. Entre los factores o elementos más importantes que lo conforman, pueden citarse los de la naturaleza (clima, aire, agua, paisaje, etc.) y la sociedad (nutrición, educación, vida social, trabajo, etc.). En este último, no se incluye por razones metodológicas y aunque es un producto cultural, el concepto tradicional de patrimonio por incluirse en el dominio de la Contabilidad Patrimonial.

Proceso contable: Conjunto de etapas lógicamente ordenadas para lograr el objetivo fijado. Entre ellas pueden mencionarse: la selección de los impactos a describir, la captación de los mismos, su clasificación, cuantificación, registro, control, exposición a través de informes sociales y análisis".

Estas apreciaciones se complementan con otra propuesta realizada a los fines de mejor precisar el contenido cognitivo y pragmático del término Contabilidad Social (Geba, 2004), donde se "utiliza la denominación de Contabilidad Social para identificar a un conjunto de conocimientos, elementos y procesos de la Disciplina Contable que, con un enfoque socioeconómico, permiten saber, de manera mayoritariamente cuantificada, los impactos sociales producidos o generados por los entes, tanto en el medio ambiente natural como en el cultural, y es útil para conocer el cumplimiento de la Res- ponsabilidad Social asumida por las organizaciones".

En función a ello se considera que la Contabilidad Patrimonial y Económico Social son especialidades de la mencionada disciplina contable que deben contar con un marco teórico que comparta un núcleo común o pegamento semántico, dentro de un denominado cinturón protector. Coincidiendo con el autor W. Chapman (1989), "La contabilidad social concierne a la recopilación, al ordenamiento, al análisis, al registro, a la síntesis y a la interpretación de los efectos que tiene la actividad de las empresas y otras entidades de la esfera económica, sobre el todo social.

Entendida a la Contabilidad Social como una especialidad de la disciplina contable, debe poseer un marco teórico conceptual relacionado con otras especialidades contables a través de los parámetros comunes de la Teoría General Contable. Es decir, como manifiesta Mario Bunge (1999) para las proposiciones de una teoría científica, un denominado tópico común o pegamento semántico. A ello agrega una relación de deductibilidad o pegamento sintáctico.

En cuanto a la relación de deductibilidad, se considera que dicho Marco Teórico General Contable debe permitir deducir la elaboración de un Modelo Teórico Contable Social General. El mismo hará posible la confección de modelos de Informes Contables Sociales. Para ello es necesario desarrollar pautas cognitivas y pragmáticas que permitan operar la recopilación, ordenamiento, análisis, registro, síntesis e interpretación de los impactos sociales producidos por los entes, a lo 
que debería adicionarse explícitamente su cuantificación, medición y evaluación metódicas. El hecho a resolver parece consistir en conocer cómo la especialidad social de la disciplina contable puede contribuir con las organizaciones para que informen sobre su Responsabilidad Social Asumida.

Se entiende que la elaboración de un Modelo Teórico Contable Social General que precise los procesos y las pautas mencionadas, así como la construcción de Modelos Contables Sociales más específicos, permitirían cumplir con tal cometido.

Generalizando todo lo antes dicho, se considera como posible y deseable "integrar las distintas especialidades contables (patrimonial, social, gerencial, pública, etc.), con sus enfoques doctrinario y normativo, los modelos en ellas generados así como sus aplicaciones, en una Estructura Teórica General o modelo más abarcativo, que permita lograr un orden primario de todo su contenido y esencia, y la incorporación sistemática de nuevos conocimientos" (Geba y Fernández, 2003).

\section{Conclusiones}

Las denominadas Organizaciones no Gubernamentales (ONGs), por su especial identidad asumen cierto grado de responsabilidad social y contribuyen, en su gran mayoría, directa o indirectamente al Desarrollo Humano Sustentable.

Para gestionarlas adecuadamente, es necesario contar con información económico financiera, pero resulta indispensable complementarla con la de tipo económico social. Esta última, al igual que la anterior, debe reunir determinados atributos o características de calidad que garantizando su sistematicidad, comparabilidad y homogeneidad, aumentan su utilidad. Para ello es necesario que surja de un adecuado "proceso contable".

Es así como día a día cobra más relevancia la especialidad contable denominada Contabilidad Social. De su desarrollo, aplicación e integración en el marco de una teoría general del conocimiento contable, depende que los informes contables socioeconómicos, tanto internos como externos, contribuyan a un gerenciamiento de las ONGs calificado como socialmente responsable, además de eficiente y eficaz. De lograrlo, el Desarrollo Humano Sustentable estará cada vez más cerca de ser una realidad.

\section{Referencias Bibliográficas}

Barbei, Alejandro y Fernández Lorenzo, Liliana (2002), "Investigación Empírica Cuantitativa en Balances Sociales Publicados", Anales de las XXIII Jornadas Universitarias de Contabilidad. Formosa, Argentina. Facultad de Administración, Economía y Negocios. Trabajo № 14, pp.1-18. Argentina.

Bunge, Mario (1999), Las ciencias sociales en discusión. Una perspectiva filosófica. Editorial Sudamericana Grafinor S.A. Argentina.

Cumbre de las Américas sobre desarrollo sostenible (1996), "Valoración ambiental de los Recursos Naturales", Documento técnico, Iniciativas. Santa Cruz, Bolivia.

Chapman, William (1989), "El desarrollo de la contabilidad social en América Latina". Anales de la V Conferencia de Facultades y Escuelas de Contaduría de América Latina y IV Congreso Lati- 
noamericano de Investigación Contable. Fundación Banco de Boston. Argentina.

Federación Argentina de Consejos Profesionales de Ciencias Económicas (2002), Resolución Técnica № 16: Marco conceptual de las normas contables profesionales. Editorial La Ley s.a. Buenos Aires.

Fernández Lorenzo, Liliana; Geba, Norma; Montes, Verónica y Schaposnik, Rosa (1998), Balance Social Cooperativo Integral. Un modelo argentino basado en la Identidad Cooperativa, Cuaderno 5 Serie Investigación del Instituto Provincial de Acción Cooperativa (IPAC). La Plata. Ministerio de la Producción y el Empleo de la Provincia de Buenos Aires.

Fernández Lorenzo, Liliana y Geba, Norma (1999), "Aportes de la Contabilidad Social al Desarrollo de las Entidades Mutuales", Anales de las XX Jornadas Universitarias de Contabilidad, Rosario, Santa Fe. Facultad de Ciencias Económicas y Estadísticas, Universidad Nacional de Rosario. pp. 65-74. Argentina.

Fernández Lorenzo, Liliana y Geba, Norma (2000a), "Responsabilidad Social Profesional Activa", Anales de las Jornadas del Cono Sur y III Jornadas Rioplatenses de Profesores de Práctica Profesional, Tomo II. 35. Morón, Argentina. Universidad de Morón, pp. 1-14.

Fernández Lorenzo, Liliana y Geba, Norma (2000b), Balance Social en Entidades Mutuales. Propuesta de un Modelo. Editorial de la Universidad Nacional de La Plata. Argentina.

Fernández Lorenzo, Liliana y Geba, Norma. Colaboradores: Cogorno, Santiago; Ravina, Natalia y Tejo Terranova, Patricia (2001). Informe final del Proyecto "Identidad cooperativa e información social" Aplicación del Balance
Social Cooperativo Integral. Principios: "Control democrático por los asociados" y "Educación, capacitación e información". Cuadernos de Economía Social. Edición Especial 35ํㅡㄹ Aniversario. Instituto Argentino de Investigaciones de Economía Social (IAIES). Buenos Aires.

Fernández Lorenzo, Liliana y Barbei, Alejandro (2004), "Contabilidad Social. Una investigación Teórica exploratoria sobre su definición y sus variables relevantes". Revista Enfoques. Contabilidad y Administración. Ed. La Ley Sociedad Editora e Impresora. pp. 47-62. Buenos Aires.

Fernández Lorenzo, Liliana y Geba, Norma. Auxiliar de Investigación Alejandro Agustín Barbei Becarios: Pamela del Buono, María Luz Modolo, Julieta Odriozola, María Laura Peiró y José Ignacio Sallenave (2003). Informe Final del proyecto de Investigación: "Información Social no económica la servicio del Mutualismo y de la Comunidad". Cuadernos de Economía Social. Año IX Número 13. Editor: Instituto Argentino de Investigaciones de Economía Social (IAIES), pp. 71 a 134. Argentina.

Ferreira Da Costa, Fernando (1984), "Las Semillas del Porvenir", Cuadernos de Economía Social. Año VI, № 17. Argentina, Instituto Argentino de Investigaciones de Economía Social (IAIES), pp. 11-30. Buenos Aires.

Gallup Argentina con la colaboración del Programa de las Naciones Unidas para el Desarrollo (1998), "Encuesta Nacional sobre Trabajo Voluntario y Donaciones en la Argentina”. Foro del Sector Social. Buenos Aires.

García Echevarría, Santiago (1976): "Balance Social de la empresa. Posibilidades y límites operativos en su planteamiento actual”. Revista Alta Dirección № 65, pp. 167. Barcelona. España. 
Geba, Norma (2001), "Un nuevo Paradigma como marco para la aplicación del método científico en la Investigación contable", Anales del 7mo. Encuentro Universitario de Investigadores del Área Contable". Universidad Nacional de Rosario. Argentina.

Geba, Norma y Fernández Lorenzo, Liliana (2002), "Reflexiones sobre el Status Epistemológico de la Contabilidad". Revista del Instituto de Estudios Contables, Año 1, № 1. La Plata. Editorial de la Universidad de La Plata, pp. 85-90. Argentina.

Geba, Norma y Fernández Lorenzo, Liliana (2003), "Investigación Teórica sobre una Posible Estructura Orgánica del Conocimiento Contable." Anales del 9no. Encuentro Nacional de Investigadores Universitarios del Área Contable. Facultad de Ciencias Económicas, Universidad Nacional de La Plata. Argentina.

Geba, Norma (2004), "Propuestas para desarrollar la Contabilidad Social y la Emisión de Balances Sociales de Entidades Lucrativas". Revista Enfoques. Contabilidad y Administración. Ed. La Ley Sociedad Editora e Impresora. pp. 30-44. Buenos Aires.

Novarese de Nieto, Teresa y Fernández Lorenzo, Liliana (1995), "Balance Social en Cooperativas de Trabajo. Propuesta para el Área Educación y Capacitación". Anales de las XVI Jornadas Universitarias de Contabilidad. Resistencia, Argentina. Universidad Nacional del Nordeste, pp. 11.1- 24. Argentina.
Osorio Meléndez, A. Hugo (2002), "Medios de Comunicación y Conflicto Social". Contribuciones 2/2002. Año XIX № 2 (74), Publicación trimestral de la Konrad-Adenauer Stifrung A.V.

Programa de las Naciones Unidas para el Desarrollo (PNUD) (2000), "Informe sobre el desarrollo humano", Barcelo, México. Ediciones Mundi - Prensa Madrid.

Programa de las Naciones Unidas para el Desarrollo (PNUD) (2003), "Informe sobre Desarrollo Humano". Http://hdr. Undp.org.reports/globa/2003/espanol/pdf/hdr03_sp_chapter_1.pdf. Cap. 1,Consulta realizada el 23-032004.

Ravina, Arturo (2000), "Formas de cooperación entre entidades de la economía social y del sector público y del privado lucrativo", 23ํㅡㄹ Congreso Internacional del CIRIEC. Montreal, Canadá. Cuadernos de Economía Social. Instituto Argentino de Investigaciones de Economía Social. Buenos Aires. 